\title{
Association of evaluated glomerular filtration rate and incident diabetes mellitus: a secondary retrospective analysis based on a Chinese Cohort Study
}

Haofei Hu

Shenzhen Second People's Hospital https://orcid.org/0000-0001-6061-6796

Mijie Guan

Shenzhen Second People's Hospital

Zhuangsen Chen

Shenzhen Second People's Hospital

Yang Wu

Shenzhen Second People's Hospital

Qijun Wan ( $\nabla$ wanqijun1234@126.com )

Department of Nephrology, The First Affiliated Hospital of Shenzhen University

\section{Research}

Keywords: Estimated glomerular filtration rate, Incident diabetes, Nonlinearity

Posted Date: July 29th, 2020

DOI: https://doi.org/10.21203/rs.3.rs-47404/v1

License: (c) (1) This work is licensed under a Creative Commons Attribution 4.0 International License.

Read Full License 
1 Association of evaluated glomerular filtration rate and incident

2 diabetes mellitus: a secondary retrospective analysis based on a

3 Chinese Cohort Study

4

Running title: eGFR and incident diabetes

6 Haofei $\mathrm{Hu}^{1,2,5 \#}$, Mijie Guan ${ }^{1,2,5 \#}$, Zhuangsen $\mathrm{Chen}^{3,4,5}$, Yang $\mathrm{Wu}^{3,4,5}$, Qijun $7 \operatorname{Wan}^{1,2,5^{*}}$

$8{ }^{1}$ Department of Nephrology, The First Affiliated Hospital of Shenzhen University, 9 Shenzhen 518035, Guangdong Province, China

$10{ }^{2}$ Department of Nephrology, Shenzhen Second People's Hospital, Shenzhen 518035,

11 Guangdong Province, China

$12{ }^{3}$ Department of Endocrinology, The First Affiliated Hospital of Shenzhen University, 13 Shenzhen 518035, Guangdong Province, China

$14{ }^{4}$ Department of Endocrinology, Shenzhen Second People's Hospital, Shenzhen 15 518035, Guangdong Province, China

$16{ }^{5}$ Shenzhen University Health Science Center, Shenzhen 518000, Guangdong Province, 17 China

18 Haofei Hu and Mijie Guan have contributed equally to this work.

$19 *$ Corresponding author

20 Qijun Wan

21 Department of Nephrology,

22 The First Affiliated Hospital of Shenzhen University, 
23 No.3002 Sungang Road, Futian District,

24 Shenzhen 518000,

25 Guangdong Province,

26 China

27 Tel:+86-755-83366388

28 E-mail: wanqijun123@126.com

29

30

31

32

33

34

35

36

37

38

39

40

41

42

43

44 


\section{Abstract}

46 Background: Previous studies have revealed that chronic kidney disease (CKD) is one of major

47 risk factors of insulin resistance and diabetes. However, there are few investigations of the

48 correlations between the estimated glomerular filtration rate (eGFR) and incident diabetes,

49 especially in Chinese population. This study was taken to explore the relationship between eGFR and incident diabetes in a large cohort in Chinese community population.

51 Methods: The present study was a retrospective cohort study. A total of 199,435 adults from Rich Healthcare Group in China, which includes all medical records for participants who received a health check from 2010 to 2016 . The target independent variable and the dependent variable were eGFR measured at baseline and incident diabetes mellitus appeared during follow-up respectively. Covariates involved in this study included age, gender, body mass index, diastolic blood pressure, systolic blood pressure, fasting plasma glucose, total cholesterol, low density lipoprotein cholesterol, high density lipoprotein cholesterol, triglyceride, alanine aminotransferase, aspartate aminotransferase, smoking and drinking status and family history of diabetes. Cox proportional-hazards regression was used to investigate the association between eGFR and incident diabetes. Generalized additive model was used to identify non-linear relationships. Additionally, we also performed a subgroup analysis. It was stated that the data had been uploaded to the DATADRYAD website.

Result: After adjusting gender, body mass index, systolic blood pressure, diastolic blood pressure,

64 fasting blood glucose, total cholesterol, low density lipoprotein cholesterol, high density lipoprotein cholesterol, triglyceride, alanine aminotransferase, aspartate aminotransferase, smoking and drinking status and family history of diabetes, result showed eGFR was negatively 
associated with incident diabetes $(\mathrm{HR}=0.987,95 \% \mathrm{CI}(0.984,0.989))$. A $\mathrm{J}$ shape relationship was detected between eGFR and incident diabetes, which had an inflection point of eGFR was $97.967 \mathrm{~mL} / \mathrm{min}^{-1} \cdot(1.73 \mathrm{~m} 2)^{-1}$. The effect sizes and the confidence intervals on the left and right sides of the inflection point were $0.999(0.994,1.004)$ and $0.977(0.974,0.981)$, respectively. Subgroup analysis showed, the stronger association can be found in the population with $\mathrm{FPG}<6.1 \mathrm{mmol} / \mathrm{L}, \mathrm{BMI}<24 \mathrm{~kg} / \mathrm{m}^{2}, \mathrm{SBP}<140 \mathrm{mmHg}, \mathrm{DBP}<90 \mathrm{mmHg}, \mathrm{HDL}$ in middle level and family history without diabetes. The same trend was also seen in men and in the population with never or ever smoking.

Conclusion: eGFR is independently associated with incident diabetes. The relationship between eGFR and incident diabetes is also non-linear. eGFR was strong negatively related to incident diabetes when eGFR is above $97.967 \mathrm{~mL} / \mathrm{min}^{-1} \cdot(1.73 \mathrm{~m} 2)^{-1}$.

Keywords: Estimated glomerular filtration rate, Incident diabetes, Nonlinearity

Background

Diabetes has become one of the most common chronic diseases all over the world. In recent decades, the prevalence of diabetes among Chinese adults has increased significantly [1]. According to a large, nationally representative survey of Chinese adults, the estimated overall prevalence of diabetes had risen to be $10.9 \%$ in 2013[2]. Therefore, it is important to explore and intervene in the risk factors of diabetes. Diabetes is a debilitating disease that may cause various complications, thereby reducing the quality of life and causing serious socio-economic impacts.

\footnotetext{
Therefore, the identification of risk factors is essential to prevent diabetes.
}

$$
\text { Patients with chronic kidney disease (CKD) and diabetes mellitus (DM) share common risk }
$$


factors[3] [4], which suggests that CKD may increase the risk of diabetes. Estimated glomerular filtration rate (eGFR) is a simpler and more applicable surrogate marker, used to describe the flow rate of filtrate through the kidney and has been widely used clinically to diagnose CKD and assess renal function[5]. A cardiovascular health study was conducted among 4680 U.S. participants without diabetes, the result[6] showed that a decreased eGFR was associated with increased insulin resistance. In their study, Mean eGFR was 72.2 (SD 17.1) ml/min per $1.73 \mathrm{~m} 2$. However, over a median follow-up of 12 years, participants with a decreased eGFR did not have an increased risk of incident diabetes. In another cohort study with 864 American participants, the result suggests that the relationship between GFR and the occurrence of diabetes was not linear, and within the upper and lower ranges of GFR, the risk of diabetes increases [7]. However, most of these studies did not perform subgroup analysis, and the relatively small sample size and regional population limited to generalizable other people. Moreover, findings from previous studies regarding the relationship between eGFR and incident diabetes was still limited in Chinese population. Therefore, this study set out to investigate whether eGFR was independently related to incident diabetes in a large cohort population across 32 sites and 11 cities in China.

\section{Methods}

\section{Data source and participants}

Date were obtained from 'DATADRYAD' database (www.Datadryad.org). This website permitted users to freely download the raw data. According to Dryad Terms of Service, we cited Dryad data package in the present study. (Dryad data package: Ying Chen, Xiao-Ping Zhang, Jie Yuan, Bo Cai, Xiao-Li Wang, Xiao-Li Wu, Yue-Hua Zhang, Xiao-Yi Zhang, Tong Yin, Xiao-Hui 
Zhu, Yun-Juan Gu1, Shi-Wei Cui, Zhi-Qiang Lu, Xiao-Ying Li(2018) Data from: Association of body mass index and age with incident diabetes in Chinese adults: a population-based cohort study. Dryad Digital Repository. http://dx.doi.org/10.1136/bmjopen-2018-021768). Variables included in the database file were as follows: age, gender, body mass index (BMI), diastolic blood pressure (DBP), systolic blood pressure (SBP), fasting plasma glucose (FPG), Triglyceride(TG), total cholesterol (TC), low density lipoprotein cholesterol(LDL-C), high density lipoprotein cholesterol (HDL-C), Serum urea nitrogen(BUN), Serum creatinine(Scr), Alanine aminotransferase(ALT), Aspartate aminotransferase(AST), smoking status, drinking status, family history of diabetes, year of follow up and censor of diabetes during follow up. In our research, we added evaluated glomerular filtration rate(eGFR), which was calculated based on age, gender and Scr according to CKD-EPI equation[8]. This new Asian modified CKD-EPI equation could lead to more accurate GFR estimation in Chinese patients with CKD in general practice, especially in the higher GFR group. Authors of the original study have waived all copyright and related ownership of these data. Therefore, we could use these data for secondary analysis without infringing on the authors' rights. As research ethics approved was obtained in the previous research, no longer needed for this secondary study.

Data were obtained from a database provided by the Rich Healthcare Group in China, and the study recruited 685,277 participants who underwent a health check and were at least 20 years old with at least two visits between 2010 and 2016 across 32 sites and 11 cities in China(Shanghai, Beijing, Nanjing, Suzhou, Shenzhen, Changzhou, Chengdu, Guangzhou, Hefei, Wuhan, Nantong). The data we obtained has been preliminarily screened, as follows:(1) no available information about weight, height, gender, fasting plasma glucose value at baseline,(2) extreme BMI values 

in eGFR which were less than means minus three standard deviation (SD) or greater than the means plus three $\operatorname{SD}(\mathrm{n}=1223)$ [10]. The final analysis included 199,435 subjects $(109,690$ male and 89,745 female ) for data analysis in our study.

\section{Study design and measurement of variables}

Researchers have obtained information (values) for our retrospective cohort study. The design of the study was documented in the original study [9]. To gave you a clearer understanding of the entire research process, we outlined the research steps here. At each visit to the health examination center, participants were asked to fill in a detailed questionnaire about demographic characteristics, lifestyle factors, personal medical history and family history of chronic diseases. Subjects were measured for height, weight and blood pressure by trained staff. Body weight was measured in light clothing with no shoes to the nearest $0.1 \mathrm{~kg}$. Height was measured to the nearest $0.1 \mathrm{~cm}$. BMI was derived from weight in kilograms divided by height in metres squared. Blood pressure was measured by standard mercury sphygmomanometers. Fasting venous blood samples were collected after at least a 10 hours fast at each visit. Scr, ALT, AST, TG, TC, LDL-C and HDL-C were measured on an autoanalyzer (Beckman 5800). Plasma glucose levels were measured by the glucose oxidase method on an autoanalyzer (Beckman 5800). The target independent variable is 
156 As this is a retrospective cohort study, it reduced the possibility of selection bias and observation

157 bias.

\section{Ascertainment of incident diabetes} self-reported diabetes during the follow-up period. Patients were censored at the date of diagnosis of diabetes or the final visit, whichever came first.

\section{Statistical analysis}

First, we dealt with the missing values of covariates. Missing continuous variables were mainly supplemented by means or medians. Since the missing values of HDL-C, LDL-C and AST were about $50 \%$, we converted them as categorical variables based on the tertiles. Besides, missing categorical variables in each covariate are considered as a independent group[11]

Next, the participants were stratified by quartiles of eGFR. Continuous variables were expressed as the means \pm standard deviations (normal distribution) or medians (quartiles) (skewed distribution), and categorical variables were expressed as a frequency or percentages. Differences between means and proportions of the groups were tested by the one-way ANOVA test for normally distributed quantitative variables, the Kruskal-Wallis test for skewed quantitative variables, and the chi-square test for categorical variables. The person-years of follow-up were

173 calculated from the date of baseline interview to the date of incident diabetes or follow-up

174 interview, whichever came first[12]. We used cumulative incidence and person-years incidence to 175 describe the incidence rate[13].Cox proportional hazard regression models were used to 176 investigate the prognostic value of eGFR on incident diabetes, and adjusted hazard ratios (HRs) 

recommendation of the STROBE statement[14], we simultaneously showed the results from unadjusted, minimally adjusted analyses and those from fully adjusted analyses. Whether the covariances were adjusted determined by the following principle: when added to this model, changed the matched hazard ratio by at least $10 \%$ [15]. To ensure the robustness of data analysis, we did a sensitivity analysis. We converted the eGFR into a categorical variable, and calculated the $\mathrm{P}$ for trend. The purpose was to verify the results of eGFR as the continuous variable and to observe the possibility of nonlinearity. We also tried to use generalized additive models (GAM) to identify non-linear relationships because eGFR was a continuous variable. If a non-linear correlation was observed, a two-piecewise linear regression model was performed to calculate the threshold effect of the eGFR on incident of diabetes in terms of the smoothing plot. When the ratio between eGFR and incident diabetes appeared obvious in a smoothed curve, the recursive method automatically calculates the inflection point, where the maximum model likelihood will be used. Moreover, the cox proportional hazard models were applied to explore robustness of the results in various subgroups (age, gender, BMI, SBP, DBP, FPG, HDL-C, LDL-C, family history of diabetes, smoking and drinking status). For continuous variable, we first converted it to a categorical 193 variable according to the clinical cut point or binary. Each stratification was adjusted for all the 194 factors, except for the stratification factor itself. The modifications and interactions of subgroups 195 were inspected by likelihood ration tests. Survival estimates and cumulative event rates were 196 compared using the Kaplan-Meier method by using the time-to-first event for each endpoint. The 197 log-rank test was used to compare the Kaplan-Meier hazard ratios (HR) for research events, and 198 their corresponding 95\% confidence intervals (CIs). 


\section{Results}

A total of 199,435 participants $(55.0 \%$ men and $45.0 \%$ women) were included in the analysis, and 142,038 .

\section{Baseline characteristics of the study participants}

Table 1 depicted the baseline characteristics of the total population and by quartiles of the

216 eGFR. We divided participants into subgroup using eGFR quartiles $(\leq 100.44,100.44-112.40$,

217 112.40-122.06, >122.06). The results showed that in highest eGFR group, participants generally

218 had lower age, BMI, blood pressure levels (including both systolic and diastolic blood pressures), 219 fasting blood glycemic, TC,TG, LDL-C, ALT, AST and lower rates of current smoker and drinker. 
with low LDL-C level. In addition, the group (eGFR $>122.06 \mathrm{~mL} / \mathrm{min}^{-1} \cdot(1.73 \mathrm{~m} 2)^{-1}$ ) had higher incidence of family history of diabetes and higher proportion of women.

223

224

\section{Incidence rate of incident diabetes}

Table 2 revealed that a total of 3919 participants developed incident diabetes. The total incidence rate of all participants was 628.73 per 100,000 person-years. Specifically, the incidence rates of the four eGFR groups were $1140.90,776.55,386.46$ and 211.19 per 100,000 person-years, respectively. Compared with the lowest eGFR group, participants with increased eGFR had a lower cumulative incidence( $\mathrm{p}<0.001$ for trend $)$. The cumulative incidence of total incident diabetes and each of the eGFR groups was 1.965 ( 1.904-2.026 ), 3.541 ( 3.379-3.703 ), 2.440 ( 2.305-2.576 ), $1.227(1.131-1.324)$ and $0.652(0.581-0.722)$, respectively.

\section{Univariate analysis}

The results of univariate analysis were shown in Table 3 . The results of univariate analysis showed that age, BMI, SBP, DBP, FPG, TG,TC, LDL, ALT, AST, family history of diabetes, smoking and drinking status were positively correlated with incident of diabetes. In contrast, HDL-C and eGFR negatively correlated with incident diabetes. We also found that women have a lower risk of developing diabetes than men.

Figure 1 showed the Kaplan-Meier curves of the cumulative hazards of diabetes incident risk stratified by eGFR categories. The risk of developing diabetes between each of the four eGFR groups was significantly different (log-rank test, $\mathrm{p}<0.0001)$. With the increase of eGFR, the cumulative risk of incident diabetes gradually decreased, rendering the top quartile group with the minimum risk of incident diabetes.

\section{The results of relationship between eGFR and incident diabetes}


244 eGFR and incident diabetes. We simultaneously showed the non-adjusted and two adjusted models

245 in Table 4. In crude model, eGFR showed negative correlation with incident diabetes $(\mathrm{HR}=0.964$,

$24695 \%$ confidence interval $(\mathrm{CI}): 0.962$ to $0.966, \mathrm{P}<0.00001)$. In minimally adjusted model (adjusted

247 gender, BMI, SBP, DBP, family history of diabetes, smoking and drinking status), the result did

248 not change significantly (HR: $0.977,95 \% \mathrm{CI}$ : 0.975-0.979). After adjusting for the full model

249 (adjusted gender, BMI, SBP, DBP, FPG, TC, TG, LDL,HDL, ALT, AST, smoking and drinking

250 status, family history of diabetes), we found the relationship still exists (HR $=0.987,95 \% \mathrm{CI}: 0.984$

251 to $0.989, \mathrm{P}<0.00001)$. The results showed that for every $1 \mathrm{~mL} / \mathrm{min}^{-1} \cdot(1.73 \mathrm{~m} 2)^{-1}$ increased in

252 eGFR, the risk of diabetes decreased by $1.3 \%$. For the purpose of sensitivity analysis, we also

253 handled eGFR as categorical variable (Quartile),the top quartile had 48.7 percent decrement of

254 diabetes risk when compared with the bottom quartile in the full model, and found that the trend

255 across the quartiles was significant( $\mathrm{P}$ for trend $<0.00001)$.

\section{The analyses of non-linear relationship}

257 In the present study, we also performed a generalized additive model (GAM) to explore the

258 non-linear relationship eGFR and incident diabetes because eGFR was a continuous variable(Fig.

259 2). We found that the relationship between eGFR and incident of diabetes was also non-linear

260 (after adjusting gender, BMI, SBP, DBP, FPG, TC, TG, LDL,HDL, ALT, AST, smoking and

261 drinking status, family history of diabetes). By using a two-piecewise linear regression model, we

262 calculated that the inflection point of eGFR was $97.967 \mathrm{~mL} / \mathrm{min}^{-1} \cdot(1.73 \mathrm{~m} 2)^{-1}$ (Log-likelihood

263 ratio test $\mathrm{P}<0.001)$. On the left of the inflection point, we observed a tiny nesitive relationship

264 between eGFR and incident diabetes(HR:0.999, 95\%CI: 0.994-1.004,P=0.6928). On the right side 
of the inflection point, however, we could found a obvious negative relationship between eGFR and incident diabetes(HR:0.977, 95\%CI: 0.974-0.981,P<0.0001).(Table 5).

\section{The results of subgroup analyses}

We used subgroup analysis to detect other potential risks in the associations between eGFR

and incident diabetes to assess the factors that might influence the results. We treated age, gender,

FPG, BMI, SBP, DBP, HDL, LDL, family history of diabetes, smoking and drinking status as the

271 stratification variables to observe the trend of effect sizes in these variables (table 6). We noted a

272 great number of interactions were observed based on our a priori specification including: gender,

273 FPG, SBP, DBP, BMI, HDL, smoking status and family history of diabetes (all P values for

274 interaction $<0.05$ ). In this study, the stronger association were detected in the population with

$275 \mathrm{FPG}<6.1 \mathrm{mmol} / \mathrm{L}, \mathrm{BMI}<24 \mathrm{~kg} / \mathrm{m}^{2}, \mathrm{SBP}<140 \mathrm{mmHg}, \mathrm{DBP}<90 \mathrm{mmHg}, \mathrm{HDL}$ in middle level and

276 family history without diabetes. Moreover, we could also found a stronger association between

277 eGFR and incident diabetes in women and in the population with never or ever smoking. In

278 contrast, the weaker association were probed in men, current smoker and the population with

$279 \mathrm{FPG} \geq 6.1 \mathrm{mmol} / \mathrm{L}, \mathrm{BMI} \geq 28 \mathrm{~kg} / \mathrm{m}^{2}, \mathrm{SBP} \geq 140 \mathrm{mmHg}, \mathrm{DBP} \geq 90 \mathrm{mmHg}, \mathrm{HDL}$ in low level and 280 family history with diabetes.

\section{Discussion}

The present retrospective cohort study showed that eGFR was negatively associated with incident diabetes after adjusting some covariates (Cox proportional hazard models). Furthermore, the trend of the effect sizes on the left and right sides of the inflection point was inconsistent

[left(HR:0.999,95\%CI: $\quad 0.994-1.004, \mathrm{P}=0.6928) ; \quad \operatorname{right}(\mathrm{HR}: \quad 0.977 ， \quad 95 \% \mathrm{CI}: \quad 0.974-0.981, \mathrm{P}$ 

between eGFR and new onset diabetes. Subgroup analysis showed a stronger association in female, never or ever smoker, and the population with $\mathrm{FPG}<6.1 \mathrm{mmol} / \mathrm{L}, \mathrm{BMI}<24 \mathrm{~kg} / \mathrm{m}^{2}, \mathrm{SBP}<140 \mathrm{mmHg}$, $\mathrm{DBP}<90 \mathrm{mmHg}, \mathrm{HDL}$ in middle level and family history without diabetes. In contrast, the weaker association were probed in male, current smoker and the population with $\mathrm{PG} \geq 6.1 \mathrm{mmol} / \mathrm{L}, \mathrm{BMI}$ $\geq 28 \mathrm{~kg} / \mathrm{m}^{2}, \mathrm{SBP} \geq 140 \mathrm{mmHg}, \mathrm{DBP} \geq 90 \mathrm{mmHg}$, HDL in low level and family history with diabetes.

A few previous studies have probed the association between eGFR and incident diabetes.

GFR and incident diabetes was not linear, which suggests that individuals in the upper and lower ranges of GFR are at increased risk of future diabetes, GFR and type 2 diabetes may share common pathogenic mechanisms. Some other studies have explored the association between CKD and incident diabetes. A prospective cohort study, focus on 1,713 American participants with reduced glomerular filtration rates and without diabetes at baseline, found that T2DM incidence rate among individuals with CKD is markedly higher than in the general population[18][18]. independent predictor of incident diabetes(adjusted HR 1.204; 95\% CI 1.11, 1.31)[19].

304 Consistently the same result that, we obtained cox proportional hazard regression model showed a negative association between eGFR and incident diabetes. Moreover, our research had a larger sample(199435) and from 32 sites and 11 cities in China, which was more representative of the

Chinese population. 
in one study with $1,337,452$ veterans conducted in United States[16], the researchers found that every $10 \mathrm{ml} / \mathrm{min} / 1.73 \mathrm{~m}^{2}$ decrease in eGFR was not associated with risk of incident diabetes $(1.00$; 1.00-1.01). A similar study in a lean, normoglycemic healthy women population in Israel, showed that in a logistic regression model adjusted for age, body mass index, socioeconomic status, smoking, baseline glucose and serum uric acid, eGFR was associated with increased risk for

314 incident diabetes $(1.02 ; 1.01-1.03)[17]$. We compared these studies mentioned above, the 315 inconsistent results may come from the following: (1) the research population is different. These studies, which were inconsistent with our findings, were targeted at Israel and America,(2) many

317 of these different conclusions do not clarify the nonlinear relationship and use different regression

318 models, (3) compared with our work, the study did not take into account the effect of SBP, DBP,

319 TC, TG, HDL,LDL, ALT, AST, Scr, drinking status and family history of diabetes, on the eGFR 320 and incident diabetes relationships when adjusting covariates. However, previous studies have 321 confirmed that these variables are related to eGFR or incident of diabetes,(4) this may be related 322 to different renal function, some studies showed that the association of eGFR and insulin 323 resistance or incident diabetes differ between different CKD stages[6][20] [21].

324 In the present study, we found using two-piecewise linear regression model to show a nonlinear 325 relation is different to that obtained by Lorenzo et al[7].In their study, they used subgroup analysis 326 stratified by GFR categories to assess a U shape relation between eGFR and risk of T2DM, they 327 found individuals in the upper and lower ranges of GFR are at increased risk of future diabetes. In 328 contrast, results of the present study indicated a $\mathrm{J}$ shape relationship on the independent association between eGFR and new onset diabetes. The difference may be caused by race, renal 330 function level and different methods for evaluating eGFR. They chose the Modification of Diet in 

used CKD-EPI equation[8] to estimate GFR, which could lead to more accurate GFR estimation

333 in Chinese patients with CKD in general practice, especially in the higher GFR group. Because of 334 the participants in our study was all with eGFR above $60 \mathrm{ml} / \mathrm{min} / 1.73 \mathrm{~m}^{2}$. Our study showed that 335 when eGFR is above $97.967 \mathrm{ml} / \mathrm{min} / 1.73 \mathrm{~m}^{2}$, the risk of diabetes decreases obviously with 336 increasing eGFR levels, these people could pay more attention to prevent the risk of diabetes. The 337 results of this study should be helpful for future studies on the establishment of diagnostic or 338 predictive models of the risk of diabetes.

In recent years, researches have elucidated the correlations between eGFR and insulin resistance. In a community-based cohort study, the result suggested that insulin sensitivity 341 measured with euglycemic clamp is independently associated with eGFR, and impaired insulin 342 sensitivity may be related to the development of renal dysfunction at an early stage, before the 343 onset of diabetes[21]. In another community-based cohort study in US older adults, researchers 344 found that lower eGFR was associated with insulin resistance. However, with lower eGFR, risks 345 of impaired glucose tolerance and incident diabetes were not increased[6]. The disturbances of 346 glucose and insulin homeostasis in CKD are complex and represent 2 two opposite effects. On the 347 one hand, CKD reduces insulin sensitivity (and increases insulin resistance) and, results in

348 beta-cell dysfunction and defective insulin secretion in advanced stages[24]. On the other hand, 349 CKD leads to decreased insulin clearance, thus prolonging its half-life[25, 26]. The balance of 350 these 2opposing forces determines the state of glucose metabolism and ultimately the risk of 351 diabetes mellitus in any individual. 

regression models.

There are still some potential limitations. Firstly, the raw data was from the Chinese population,

371 concerned in this study. Finally, although we have adjusted for a number of confounding factors to

372 the possible influences, residual confounding may exit and further investigations are needed.

\section{Conclusion}

374 eGFR is independently associated with incident diabetes. The relationship between eGFR and 
incident diabetes is also non-linear. eGFR is obvious negatively related with incident diabetes when eGFR is above $97.967 \mathrm{~mL} / \mathrm{min}^{-1} \cdot(1.73 \mathrm{~m} 2)^{-1}$. In addition, the stronger association of eGFR and incident diabetes were detected in female, never or ever smoker, and the population with $\mathrm{FPG}<6.1 \mathrm{mmol} / \mathrm{L}, \mathrm{BMI}<24 \mathrm{~kg} / \mathrm{m}^{2}, \mathrm{SBP}<140 \mathrm{mmHg}, \mathrm{DBP}<90 \mathrm{mmHg}, \mathrm{HDL}$ in middle level and family history without diabetes.

\section{Abbreviations}

BMI, Body mass index; SBP, Systolic blood pressure; DBP, Diastolic blood pressure; FPG; Fasting plasma glucose; Scr, Serum creatinine; eGFR, evaluated glomerular filtration rate; TC, Total cholesterol; TG , Triglyceride; HDL-C, High-density lipoprotein cholesterol; LDL-C, Low-density lipid cholesterol; ALT, Alanine aminotransferase; AST, Aspartate aminotransferase; GAM, Generalized additive models; CKD, Chronic kidney disease; T2DM, Type 2 diabetes mellitus; HR, hazard ratios; CI, Confidence intervals.

\section{Authors' contributions}

Haofei HU and Mijie GUAN contributed to the study concept and design, researched and interpreted the data and drafted the manuscript. Zhuangsen CHEN and Yang WU oversaw the progress of the project, contributed to the discussion and reviewed the manuscript. Qijun WAN revised the manuscript. Haofei HU are the guarantors of this work and, as such, had full access to all the data in the study and take responsibility for the integrity of the data and the accuracy of the data analysis .All authors read and approved the final manuscript.

\section{Acknowledgements}

Not applicable 
398 The authors declare that they have no competing interests

399

400

401

402

403

404

405

406

407

408

409

410

411

412

413

414

415

416

417

418

419

420

421

422

423

424

425

\section{Availability of data and materials}

Data can be downloaded from 'DATADRYAD' database (www.Datadryad.org).

\section{Consent for publication}

Not applicable.

\section{Ethics approval and consent to participate}

In the previously published article[9], Ying Chen, et al. has clearly stated that: the study was conducted in accordance with the Declaration of Helsinki. Informed consent was obtained from all Participants.

\section{Funding}

This study was supported by Discipline Construction Ability Enhancement Project of Shenzhen Municipal Health Commission (SZXJ2017031). 年

(1)

(1)

\section{References:}

1. Weng J, Ji L, Jia W, Lu J, Zhou Z, Zou D, Zhu D, Chen L, Chen L, Guo L et al: Standards of care for type 2 diabetes in China. Diabetes/Metabolism Research and Reviews 2016, 32(5):442-458.

2. Wang L, Gao P, Zhang M, Huang Z, Zhang D, Deng Q, Li Y, Zhao Z, Qin X, Jin D et al: Prevalence and Ethnic Pattern of Diabetes and Prediabetes in China in 2013. JAMA 2017, 317(24):2515.

3. Aschner P: Metabolic syndrome as a risk factor for diabetes. Expert Review of Cardiovascular Therapy 2010, 8(3):407-412.

4. Laguardia HA, Hamm LL, Chen J: The metabolic syndrome and risk of chronic kidney disease: pathophysiology and intervention strategies. J Nutr Metab 2012, 2012:652608.

5. Warnock DG: Estimated Glomerular Filtration Rate: Fit for What Purpose? NEPHRON 2016, 134(1):43-49.

6. Pham H, Robinson-Cohen C, Biggs ML, Ix JH, Mukamal KJ, Fried LF, Kestenbaum B, Siscovick DS, de Boer IH: Chronic kidney disease, insulin resistance, and incident diabetes in older adults. 
Clin J Am Soc Nephrol 2012, 7(4):588-594.

7. Lorenzo C, Nath SD, Hanley AJ, Abboud HE, Gelfond JA, Haffner SM: Risk of type 2 diabetes among individuals with high and low glomerular filtration rates. DIABETOLOGIA 2009, 52(7):1290-1297.

8. Wang J, Xie P, Huang J, Qu Y, Zhang F, Wei L, Fu P, Huang X: The new Asian modified CKD-EPI equation leads to more accurate GFR estimation in Chinese patients with CKD. INT UROL NEPHROL 2016, 48(12):2077-2081.

9. Chen Y, Zhang X, Yuan J, Cai B, Wang X, Wu X, Zhang Y, Zhang X, Yin T, Zhu X et al: Association of body mass index and age with incident diabetes in Chinese adults: a population-based cohort study. BMJ OPEN 2018, 8(9):e21768.

10. Zhang N, Hu X, Zhang Q, Bai P, Cai M, Zeng TS, Zhang JY, Tian SH, Min J, Huang HT et al: Non-high-density lipoprotein cholesterol: High-density lipoprotein cholesterol ratio is an independent risk factor for diabetes mellitus: Results from a population-based cohort study. $J$ DIABETES 2018, 10(9):708-714.

11. Erviti J, Alonso Á, Oliva B, Gorricho J, López A, Timoner J, Huerta C, Gil M, De Abajo F: Oral bisphosphonates are associated with increased risk of subtrochanteric and diaphyseal fractures in elderly women: a nested case - control study. BMJ OPEN 2013, 3(1):e2091.

12. Guo C, Qin P, Li Q, Zhang D, Tian G, Liu D, Liu L, Cheng C, Chen X, Qie R et al: Association between mean arterial pressure and risk of type 2 diabetes mellitus: The Rural Chinese Cohort Study. PRIM CARE DIABETES 2020.

13. Qin H, Chen Z, Zhang Y, Wang L, Zhang Y: Triglyceride to high density lipoprotein cholesterol ratio is associated with incident diabetes in male: A retrospective study in Chinese. $J$ DIABETES INVEST 2019.

14. Yokoyama M, Watanabe T, Otaki Y, Takahashi H, Arimoto T, Shishido T, Miyamoto T, Konta T, Shibata Y, Daimon $\mathrm{M}$ et al: Association of the Aspartate Aminotransferase to Alanine Aminotransferase Ratio with BNP Level and Cardiovascular Mortality in the General Population: The Yamagata Study 10-Year Follow-Up. DIS MARKERS 2016, 2016:1-9.

15. Vandenbroucke JP, von Elm E, Altman DG, Gøtzsche PC, Mulrow CD, Pocock SJ, Poole C, Schlesselman JJ, Egger M: Strengthening the Reporting of Observational Studies in Epidemiology (STROBE): Explanation and elaboration. INT J SURG 2014, 12(12):1500-1524.

16. Xie Y, Bowe B, Li T, Xian H, Yan Y, Al-Aly Z: Higher blood urea nitrogen is associated with increased risk of incident diabetes mellitus. KIDNEY INT 2018, 93(3):741-752.

17. Shani M, Vinker S, Dinour D, Leiba M, Twig G, Holtzman EJ, Leiba A: High Normal Uric Acid Levels Are Associated with an Increased Risk of Diabetes in Lean, Normoglycemic Healthy Women. J Clin Endocrinol Metab 2016, 101(10):3772-3778.

18. Jepson C, Hsu JY, Fischer MJ, Kusek JW, Lash JP, Ricardo AC, Schelling JR, Feldman HI, Appel LJ, Feldman HI et al: Incident Type 2 Diabetes Among Individuals With CKD: Findings From the Chronic Renal Insufficiency Cohort (CRIC) Study. AM J KIDNEY DIS 2019, 73(1):72-81.

19. Lin CY, Hsieh MC, Kor CT, Hsieh YP: Association and risk factors of chronic kidney disease and incident diabetes: a nationwide population-based cohort study. DIABETOLOGIA 2019, 62(3):438-447.

20. Wu PP, Kor CT, Hsieh MC, Hsieh YP: Association between End-Stage Renal Disease and Incident Diabetes Mellitus-A Nationwide Population-Based Cohort Study. J CLIN MED 2018, 

7(10). 21. Nerpin E, Riserus U, Ingelsson E, Sundstrom J, Jobs M, Larsson A, Basu S, Arnlov J: Insulin sensitivity measured with euglycemic clamp is independently associated with glomerular filtration rate in a community-based cohort. DIABETES CARE 2008, 31(8):1550-1555.

22. Levey AS, Coresh J, Balk E, Kausz AT, Levin A, Steffes MW, Hogg RJ, Perrone RD, Lau J, Eknoyan G: National Kidney Foundation practice guidelines for chronic kidney disease: evaluation, classification, and stratification. ANN INTERN MED 2003, 139(2):137-147.

23. Levey AS, Bosch JP, Lewis JB, Greene T, Rogers N, Roth D: A more accurate method to estimate glomerular filtration rate from serum creatinine: a new prediction equation. Modification of Diet in Renal Disease Study Group. ANN INTERN MED 1999, 130(6):461-470.

24. Siew ED, Ikizler TA: Insulin resistance and protein energy metabolism in patients with advanced chronic kidney disease. Semin Dial 2010, 23(4):378-382.

25. Koppe L, Pelletier CC, Alix PM, Kalbacher E, Fouque D, Soulage CO, Guebre-Egziabher F: Insulin resistance in chronic kidney disease: new lessons from experimental models. Nephrol Dial Transplant 2014, 29(9):1666-1674.

26. Katz AI, Rubenstein AH: Metabolism of proinsulin, insulin, and C-peptide in the rat. $J$ CLIN INVEST 1973, 52(5):1113-1121.

27. Alberti KG, Zimmet PZ: Definition, diagnosis and classification of diabetes mellitus and its complications. Part 1: diagnosi $s$ and classification of diabetes mellitus provisional report of a WHO consultation. Diabet Med 1998, 15(7):539-553.

(1)

.

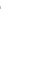

\section{3}

(1)

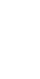
(

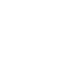




\begin{tabular}{|c|c|c|c|c|c|}
\hline eGFR (quartile) & $\mathrm{Q} 1(\leq 100.44)$ & $\begin{array}{l}\text { Q2(100.44 to } \\
112.40)\end{array}$ & $\begin{array}{l}\text { Q3(112.40 to } \\
122.06)\end{array}$ & Q4(>112.06) & P-value \\
\hline Participants & 49843 & 49874 & 49858 & 49860 & \\
\hline AGE(years) & $52.91 \pm 14.33$ & $45.24 \pm 10.58$ & $38.27 \pm 6.57$ & $31.83 \pm 4.54$ & $<0.001$ \\
\hline GENDER & & & & & $<0.001$ \\
\hline Male & $33460(67.13 \%)$ & $29983(60.12 \%)$ & $26243(52.64 \%)$ & $20004(40.12 \%)$ & \\
\hline Female & $16383(32.87 \%)$ & $19891(39.88 \%)$ & $23615(47.36 \%)$ & $29856(59.88 \%)$ & \\
\hline $\operatorname{BMI}\left(\mathrm{kg} / \mathrm{m}^{2}\right)$ & $24.12 \pm 3.14$ & $23.63 \pm 3.19$ & $23.05 \pm 3.29$ & $22.14 \pm 3.41$ & $<0.001$ \\
\hline FPG(mmol/L) & $5.05 \pm 0.64$ & $4.96 \pm 0.63$ & $4.85 \pm 0.59$ & $4.79 \pm 0.55$ & $<0.001$ \\
\hline SBP(mmHg) & $124.66 \pm 18.11$ & $120.19 \pm 16.17$ & $116.39 \pm 14.72$ & $114.46 \pm 14.04$ & $<0.001$ \\
\hline DBP(mmHg) & $76.85 \pm 11.21$ & $75.41 \pm 10.89$ & $73.15 \pm 10.38$ & $71.11 \pm 9.69$ & $<0.001$ \\
\hline $\operatorname{eGFR}\left(m L / \min ^{-1} \cdot(1.73 \mathrm{~m} 2)^{-1}\right)$ & $89.71 \pm 8.51$ & $106.68 \pm 3.44$ & $117.37 \pm 2.77$ & $127.98 \pm 4.65$ & $<0.001$ \\
\hline $\mathrm{TC}(\mathrm{mmol} / \mathrm{L})$ & $4.95 \pm 0.92$ & $4.83 \pm 0.90$ & $4.63 \pm 0.84$ & $4.43 \pm 0.82$ & $<0.001$ \\
\hline $\mathrm{TG}(\mathrm{mmol} / \mathrm{L})$ & $1.28(0.90-1.88)$ & $1.16(0.80-1.73)$ & $1.02(0.71-1.51)$ & $0.88(0.63-1.24)$ & $<0.001$ \\
\hline HDL-C(tertile) & & & & & $<0.001$ \\
\hline Low & $11327(22.73 \%)$ & $10803(21.66 \%)$ & $9350(18.75 \%)$ & $6609(13.26 \%)$ & \\
\hline Medium & $11190(22.45 \%)$ & $10066(20.18 \%)$ & $8959(17.97 \%)$ & $7339(14.72 \%)$ & \\
\hline High & $10106(20.28 \%)$ & $10186(20.42 \%)$ & $9755(19.57 \%)$ & $9343(18.74 \%)$ & \\
\hline Not recorded & $17220(34.55 \%)$ & $18819(37.73 \%)$ & $21794(43.71 \%)$ & $26569(53.29 \%)$ & \\
\hline LDL-C(tertile) & & & & & $<0.001$ \\
\hline Low & $8381(16.81 \%)$ & $9057(18.16 \%)$ & $10354(20.77 \%)$ & $10560(21.18 \%)$ & \\
\hline Medium & 10951 (21.97\%) & $10510(21.07 \%)$ & $9512(19.08 \%)$ & $7695(15.43 \%)$ & \\
\hline High & $14380(28.85 \%)$ & $11556(23.17 \%)$ & $8165(16.38 \%)$ & $5000(10.03 \%)$ & \\
\hline Not recorded & $16131(32.36 \%)$ & $18751(37.60 \%)$ & $21827(43.78 \%)$ & $26605(53.36 \%)$ & \\
\hline $\operatorname{ALT}(\mathbf{U} / \mathbf{L})$ & $19.60(14.50-28.00)$ & $19.20(14.00-29.00)$ & $18.00(12.50-28.00)$ & $15.20(11.10-24.00)$ & $<0.001$ \\
\hline AST(tertile) & & & & & $<0.001$ \\
\hline Low & $5371(10.78 \%)$ & $6413(12.86 \%)$ & $7421(14.88 \%)$ & $8710(17.47 \%)$ & \\
\hline Medium & $8037(16.12 \%)$ & $7400(14.84 \%)$ & $6612(13.26 \%)$ & $6176(12.39 \%)$ & \\
\hline High & $9382(18.82 \%)$ & $7399(14.84 \%)$ & $6296(12.63 \%)$ & $4987(10.00 \%)$ & \\
\hline Not recorded & $27053(54.28 \%)$ & $28662(57.47 \%)$ & $29529(59.23 \%)$ & 29987 (60.14\%) & \\
\hline Smoking status & & & & & $<0.001$ \\
\hline Never smoker & $9741(19.54 \%)$ & $9821(19.69 \%)$ & $11386(22.84 \%)$ & $12657(25.39 \%)$ & \\
\hline Ever smoker & $661(1.33 \%)$ & $591(1.18 \%)$ & $682(1.37 \%)$ & $530(1.06 \%)$ & \\
\hline Current smoker & $3555(7.13 \%)$ & $3628(7.27 \%)$ & $2571(5.16 \%)$ & $1574(3.16 \%)$ & \\
\hline Not recorded & $35886(72.00 \%)$ & $35834(71.85 \%)$ & $35219(70.64 \%)$ & $35099(70.40 \%)$ & \\
\hline Drinking status & & & & & $<0.001$ \\
\hline Never drinker & $11201(22.47 \%)$ & $11198(22.45 \%)$ & $12067(24.20 \%)$ & $13026(26.13 \%)$ & \\
\hline Ever drinker & $2301(4.62 \%)$ & $2419(4.85 \%)$ & $2291(4.60 \%)$ & $1612(3.23 \%)$ & \\
\hline Current drinker & $455(0.91 \%)$ & $423(0.85 \%)$ & $281(0.56 \%)$ & $123(0.25 \%)$ & \\
\hline Not recorded & $35886(72.00 \%)$ & $35834(71.85 \%)$ & $35219(70.64 \%)$ & $35099(70.40 \%)$ & \\
\hline Family history of diabetes & & & & & $<0.001$ \\
\hline No & $49025(98.36 \%)$ & $48807(94.86 \%)$ & $48587(97.45 \%)$ & $48820(97.91 \%)$ & \\
\hline YES & $818(1.64 \%)$ & $1067(2.14 \%)$ & $1271(2.55 \%)$ & $1040(2.09 \%)$ & \\
\hline
\end{tabular}


Values are $\mathrm{n}(\%)$, mean $\pm \mathrm{SD}$ or medians (quartiles)

505 BMI, body mass index; SBP, Systolic blood pressure; DBP, Diastolic blood pressure; FPG, fasting plasma glucose; TC, Total cholesterol; TG,Triglyceride; LDL-C, Low-density lipid cholesterol; HDL-C, high-density lipoprotein cholesterol; ALT, Alanine aminotransferase; AST, Aspartate aminotransferase; eGFR evaluated glomerular filtration rate Scr,Serum creatinine.

509

510

511

512

513

514

515

516

517

518

519

520

521

522

523

524

525

526

527

528

529

530

531

532

533

534

535

536

537

538

539

540

541

542

543

544

545

546 
548 Table 2 Incidence rate of incident diabetes

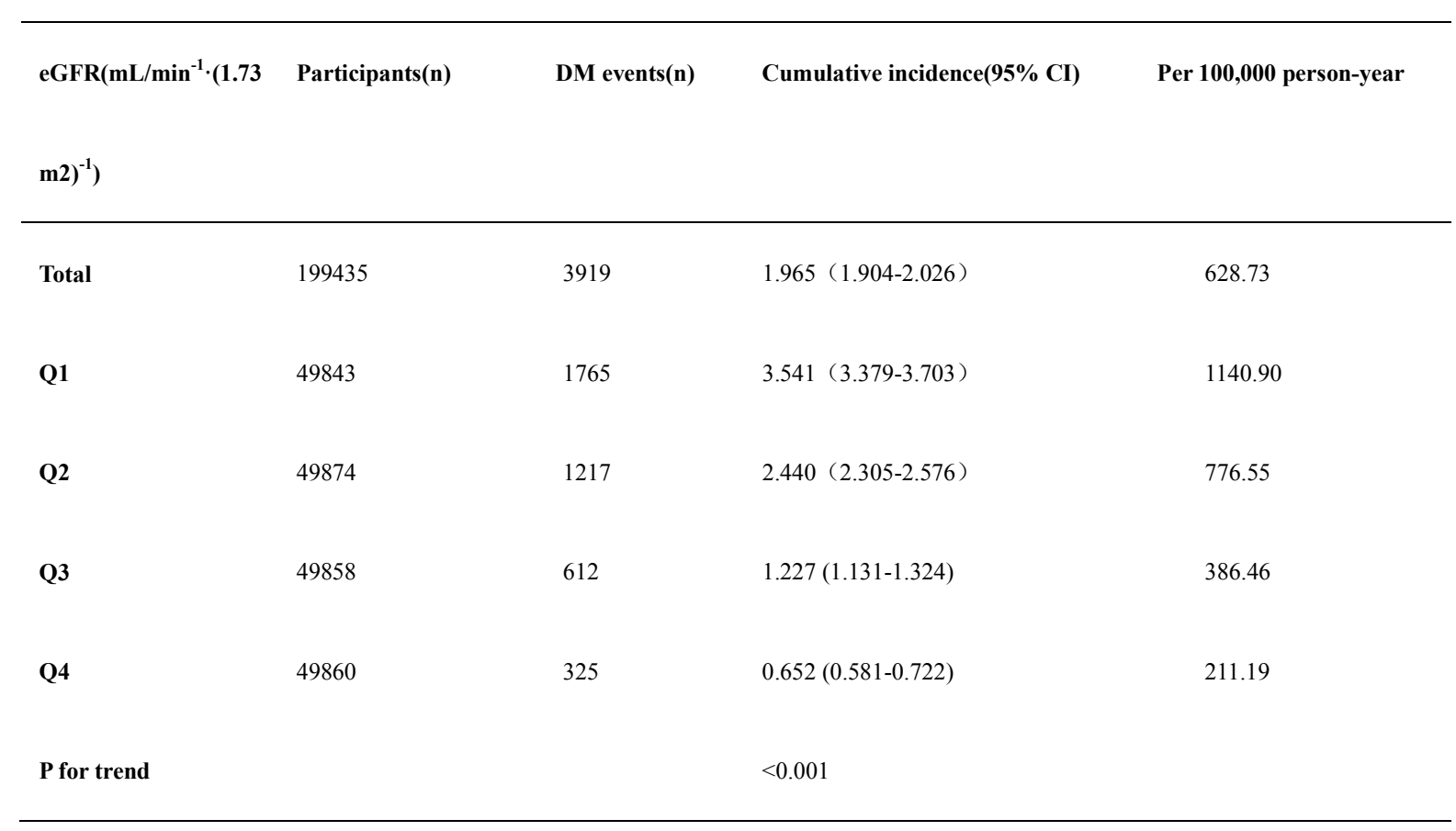

MAP, Mean arterial pressure ; CI, confidence interval; DM, diabetes mellitus.

550

551

552

553

554

555

556

557

558

559

560

561

562

563

564

565

566

567

568

569

570

571 
Table 3 The results of univariate analysis

\begin{tabular}{|c|c|c|c|}
\hline & Statistics & HR $(95 \% C I)$ & $P$ value \\
\hline Age (years) & $42.064 \pm 12.530$ & $1.066(1.064,1.069)$ & $<0.00001$ \\
\hline \multicolumn{4}{|l|}{ Gender } \\
\hline Male & $109690(55.000 \%)$ & Ref. & \\
\hline Female & $89745(45.000 \%)$ & $0.476(0.444,0.511)$ & $<0.00001$ \\
\hline $\operatorname{BMI}\left(\mathrm{Kg} / \mathbf{m}^{2}\right)$ & $23.235 \pm 3.339$ & $1.237(1.228,1.246)$ & $<0.00001$ \\
\hline SBP(mmHg) & $118.922 \pm 16.308$ & $1.039(1.037,1.041)$ & $<0.00001$ \\
\hline DBP(mmHg) & $74.126 \pm 10.782$ & $1.047(1.044,1.049)$ & $<0.00001$ \\
\hline $\operatorname{eGFR}\left(\mathrm{mL} / \mathrm{min}^{-1} \cdot(1.73 \mathrm{~m} 2)^{-1}\right)$ & $110.438 \pm 15.109$ & $0.964(0.962,0.966)$ & $<0.00001$ \\
\hline FPG(mmol/L) & $4.913 \pm 0.612$ & $10.494(10.035,10.974)$ & $<0.00001$ \\
\hline $\mathrm{TC}(\mathrm{mmol} / \mathrm{L})$ & $4.713 \pm 0.892$ & $1.428(1.386,1.471)$ & $<0.00001$ \\
\hline TG(mmol/L) & $1.338 \pm 1.025$ & $1.264(1.252,1.276)$ & $<0.00001$ \\
\hline \multicolumn{4}{|l|}{ HDL-C(mmol/L) } \\
\hline Low & $38089(19.098 \%)$ & Ref. & \\
\hline Medium & $37554(18.830 \%)$ & $0.836(0.763,0.917)$ & 0.00013 \\
\hline High & $39390(19.751 \%)$ & $0.735(0.669,0.809)$ & $<0.00001$ \\
\hline Not recorded & $84402(42.321 \%)$ & $0.585(0.541,0.633)$ & $<0.00001$ \\
\hline \multicolumn{4}{|l|}{ LDL-C(mmol/L) } \\
\hline Low & $38352(19.230 \%)$ & Ref. & \\
\hline Medium & $38668(19.389 \%)$ & $1.157(1.046,1.281)$ & 0.00462 \\
\hline High & $39101(19.606 \%)$ & $1.701(1.548,1.868)$ & $<0.00001$ \\
\hline Not recorded & $83314(41.775 \%)$ & $0.824(0.751,0.903)$ & 0.00004 \\
\hline $\operatorname{ALT}(\mathbf{U} / \mathbf{L})$ & $23.973 \pm 22.031$ & $1.005(1.004,1.005)$ & $<0.00001$ \\
\hline \multicolumn{4}{|l|}{$\operatorname{AST}(\mathbf{U} / \mathbf{L})$} \\
\hline Low & $27915(13.997 \%)$ & Ref. & \\
\hline Medium & $28225(14.152 \%)$ & $1.419(1.235,1.630)$ & $<0.00001$ \\
\hline High & $28064(14.072 \%)$ & $2.775(2.447,3.147)$ & $<0.00001$ \\
\hline Not recorded & $115231(57.779 \%)$ & $1.380(1.228,1.551)$ & $<0.00001$ \\
\hline \multicolumn{4}{|l|}{ Smoking status } \\
\hline Never smoker & $43605(21.864 \%)$ & Ref. & \\
\hline Ever smoker & $2464(1.235 \%)$ & $1.884(1.483,2.394)$ & $<0.00001$ \\
\hline Current smoker & $11328(5.680 \%)$ & $2.308(2.034,2.618)$ & $<0.00001$ \\
\hline Not recorded & $142038(71.220 \%)$ & $1.356(1.244,1.477)$ & $<0.00001$ \\
\hline \multicolumn{4}{|l|}{ Drinking stauts } \\
\hline Never drinker & $47492(23.813 \%)$ & Ref. & \\
\hline Ever drinker & $8623(4.324 \%)$ & $1.037(0.883,1.218)$ & 0.65585 \\
\hline Current drinker & $1282(0.643 \%)$ & $2.179(1.620,2.931)$ & $<0.00001$ \\
\hline Not recorded & $142038(71.220 \%)$ & $1.072(0.993,1.156)$ & 0.07433 \\
\hline \multicolumn{4}{|l|}{ Family history of diabetes } \\
\hline No & $195239(97.896 \%)$ & Ref. & \\
\hline Yes & $4196(2.104 \%)$ & $1.695(1.448,1.984)$ & $<0.00001$ \\
\hline
\end{tabular}


575 Fig1.Kaplan-Meier event-free survival curve.

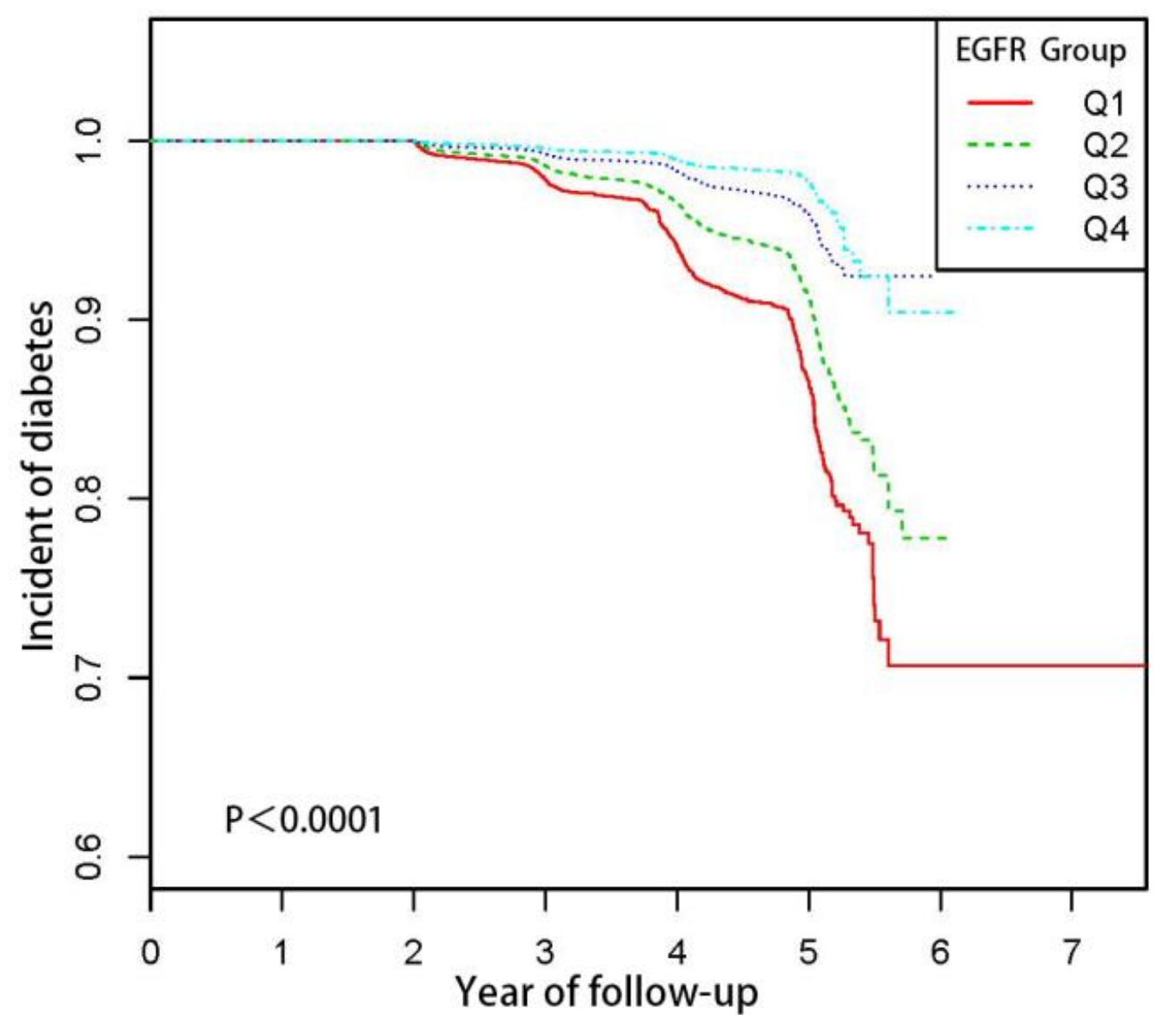

576

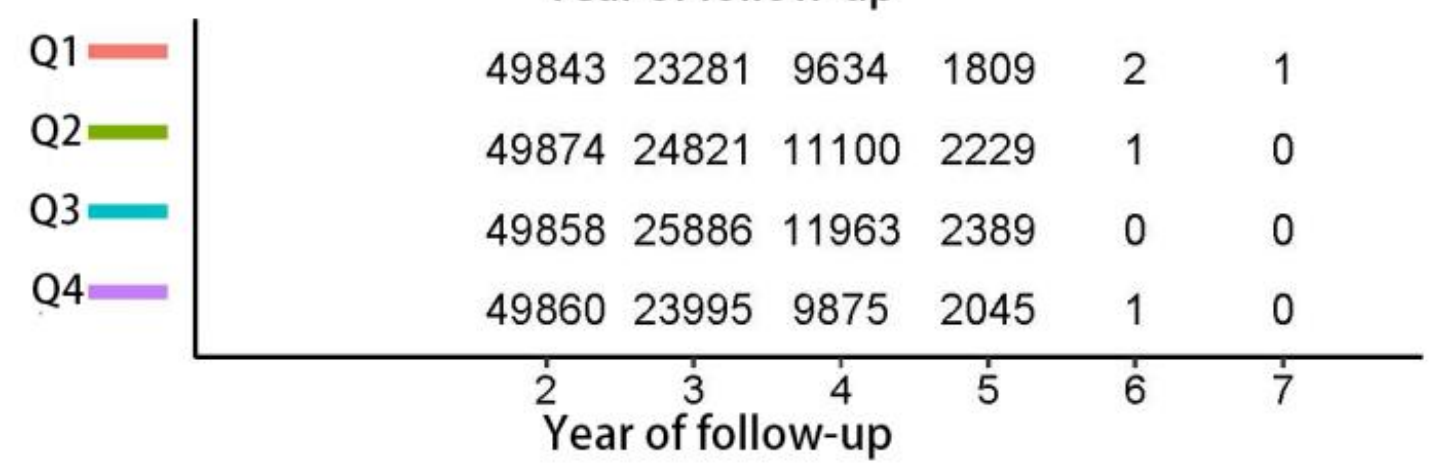

577 Fig 1.Kaplan-Meier event-free survival curve. Kaplan-Meier analysis of incident of diabetes based on eGFR quartiles (log-rank, P < $5780.0001)$.

579 
Table 4 Relationship eGFR and the incident of diabetes in different models

\begin{tabular}{clll}
\hline Exposure & Crude model $($ HR,95\%CI,P) & Adjust I $($ HR,95\%CI,P) & Adjust II $($ HR,95\%CI,P) \\
\hline eGFR & $0.964(0.962,0.966)<0.00001$ & $0.977(0.975,0.979)<0.00001$ & $0.987(0.984,0.989)<0.00001$ \\
eGFR(quartile) & & & \\
Q1 & 1.0 & 1.0 & 1.0 \\
Q2 & $0.633(0.589,0.681)<0.00001$ & $0.765(0.711,0.824)<0.00001$ & $0.824(0.765,0.888)<0.00001$ \\
Q3 & $0.306(0.279,0.336)<0.00001$ & $0.444(0.405,0.488)<0.00001$ & $0.611(0.555,0.672)<0.00001$ \\
Q4 & $0.181(0.160,0.203)<0.00001$ & $0.314(0.278,0.354)<0.00001$ & $0.513(0.453,0.581)<0.00001$ \\
P for trend & $<0.00001$ & $<0.00001$ & $<0.00001$ \\
\hline
\end{tabular}

Crude model: we did not adjust other covariates. drinking status. 
617 Fig2. The non-linear relationship between eGFR and incident of diabetes.

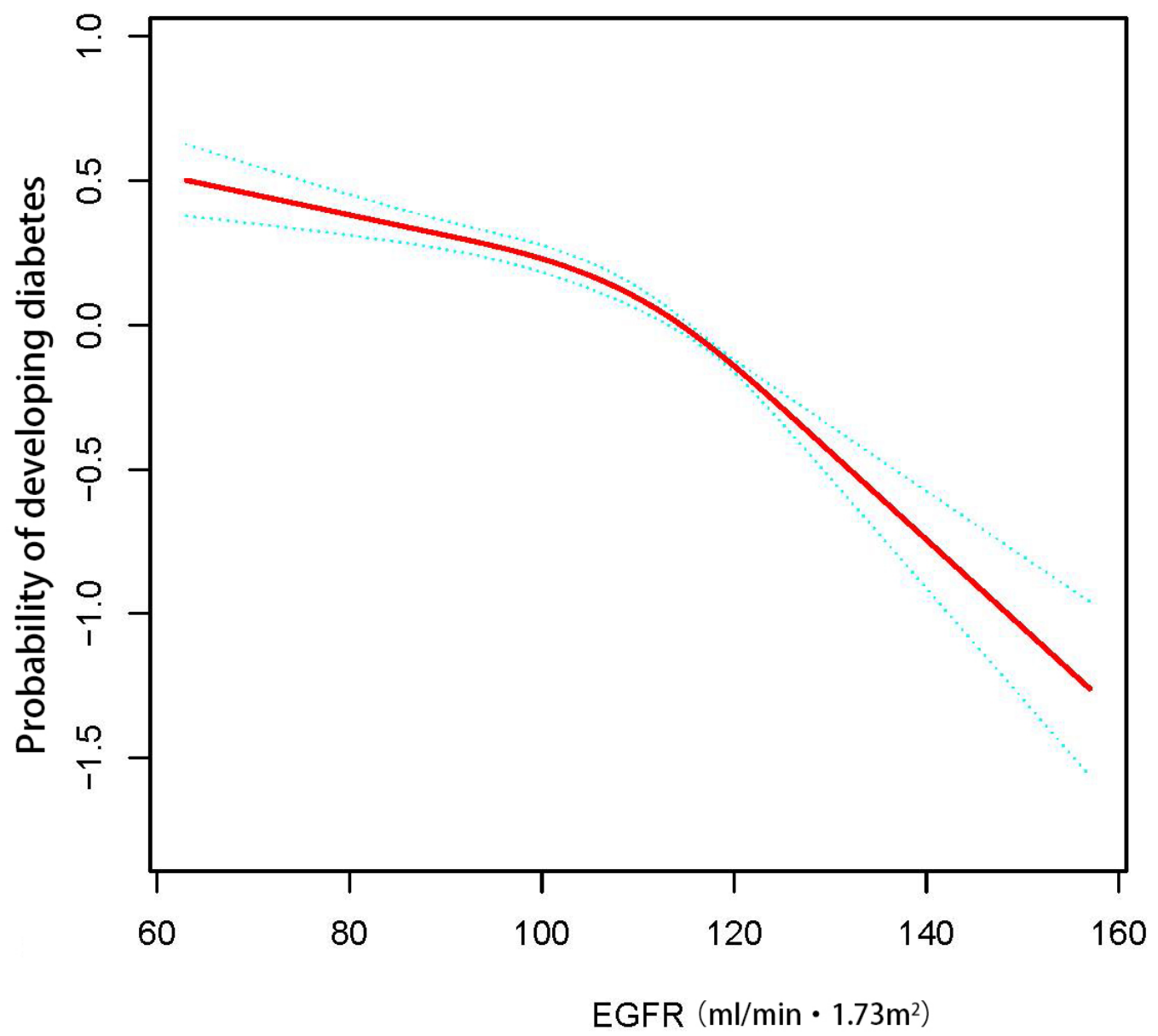

618

Fig 2.The non-linear relationship between eGFR and incident of diabetes. A non-linear relationship between them was detected after 
633

634 Table 5 The result of two-piecewise linear regression model

\begin{tabular}{|c|c|c|}
\hline & \multicolumn{2}{|c|}{ incident of diabetes (HR,95\%CI, P ) } \\
\hline Fitting model by standard linear regression & $0.987(0.984,0.989)$ & $<0.0001$ \\
\hline \multicolumn{3}{|l|}{ Fitting model by two-piecewise linear regression } \\
\hline Inflection point of eGFR & 97.769 & \\
\hline$\leq 97.769$ & $0.999(0.994,1.004)$ & 0.6928 \\
\hline$>97.769$ & $0.977(0.974,0.981)$ & $<0.0001$ \\
\hline $\mathrm{P}$ for log likelihood ratio test & $<0.001$ & \\
\hline
\end{tabular}

635

CI: Confidence interval

636 We adjusted gender, BMI, SBP, DBP, FPG, TC, TG, HDL-C, LDL-C, ALT, AST, family history of diabetes, smoking and drinking status

637

638

639

640

641

642

643

644

645

646

647

648

649

650

651

652

653

654

655

656

657

658

659

660

661

662

663

664

665

666

667

668

669 
671 Table 6 Effect size of eGFR on incident diabetes in prespecified and exploratory subgroups

\begin{tabular}{|c|c|c|c|c|}
\hline Characteristic & No of participants & HR $\quad(95 \% C I)$ & $P$ value & $P$ for interacion \\
\hline Age(years) & & & & 0.4915 \\
\hline 20 to $<30$ & 26726 & $0.999(0.978,1.019)$ & 0.8890 & \\
\hline 30 to $<40$ & 78227 & $1.011(1.003,1.019)$ & 0.0098 & \\
\hline 40 to $<50$ & 42909 & $1.005(0.998,1.012)$ & 0.1342 & \\
\hline 50 to $<60$ & 28388 & $1.002(0.997,1.008)$ & 0.3874 & \\
\hline 60 to $<70$ & 16795 & $1.004(0.997,1.010)$ & 0.2783 & \\
\hline$\geq 70$ & 6390 & $1.010(1.001,1.020)$ & 0.0389 & \\
\hline Gender & & & & 0.0013 \\
\hline Male & 109690 & $0.989(0.987,0.992)$ & $<0.0001$ & \\
\hline Female & 89745 & $0.981(0.977,0.985)$ & $<0.0001$ & \\
\hline BMI $\left(\mathrm{Kg} / \mathbf{m}^{2}\right)$ & & & & $<0.0001$ \\
\hline$<18.5$ & 11367 & $0.968(0.946,0.990)$ & 0.0045 & \\
\hline$\geq 18.5,<24$ & 109984 & $0.980(0.976,0.984)$ & $<0.0001$ & \\
\hline$\geq 24,<28$ & 61047 & $0.986(0.983,0.989)$ & $<0.0001$ & \\
\hline$\geq \mathbf{2 8}$ & 17037 & $0.998(0.994,1.002)$ & 0.4033 & \\
\hline FPG $(\mathrm{mmol} / \mathrm{L})$ & & & & $<0.0001$ \\
\hline$<6.1$ & 192704 & $0.978(0.975,0.981)$ & $<0.0001$ & \\
\hline$\geq 6.1$ & 6731 & $0.994(0.991,0.997)$ & 0.0004 & \\
\hline HDL-C(mmol/L) & & & & 0.0204 \\
\hline Low & 38089 & $0.991(0.988,0.995)$ & $<0.0001$ & \\
\hline Medium & 37554 & $0.983(0.978,0.988)$ & $<0.0001$ & \\
\hline High & 39390 & $0.987(0.982,0.992)$ & $<0.0001$ & \\
\hline Not recorded & 84402 & $0.984(0.981,0.988)$ & $<0.0001$ & \\
\hline LDL-C(mmol/L) & & & & 0.0560 \\
\hline Low & 38352 & $0.984(0.979,0.988)$ & $<0.0001$ & \\
\hline Medium & 38668 & $0.990(0.985,0.994)$ & $<0.0001$ & \\
\hline High & 39101 & $0.989(0.985,0.993)$ & $<0.0001$ & \\
\hline Not recorded & 83314 & $0.984(0.980,0.988)$ & $<0.0001$ & \\
\hline Smoking status & & & & 0.0038 \\
\hline Never smoker & 43605 & $0.983(0.977,0.988)$ & $<0.0001$ & \\
\hline Ever smoker & 2464 & $0.979(0.963,0.996)$ & 0.0175 & \\
\hline Current smoker & 11328 & $0.999(0.991,1.007)$ & 0.7830 & \\
\hline Not recorded & 142038 & $0.986(0.984,0.989)$ & $<0.0001$ & \\
\hline Drinking status & & & & 0.7418 \\
\hline Never drinker & 47492 & $0.986(0.981,0.990)$ & $<0.0001$ & \\
\hline Ever drinker & 8623 & $0.987(0.976,0.998)$ & 0.0216 & \\
\hline Current drinker & 1282 & $1.001(0.974,1.028)$ & 0.9536 & \\
\hline Not recorded & 142038 & $0.986(0.984,0.989)$ & $<0.0001$ & \\
\hline Family history of diabetes & & & & 0.0416 \\
\hline No & 195239 & $0.986(0.984,0.988)$ & $<0.0001$ & \\
\hline Yes & 4196 & $0.998(0.987,1.009)$ & 0.6964 & \\
\hline
\end{tabular}




\begin{tabular}{lcccc}
\hline SBP & & & 0.0011 \\
$<\mathbf{1 4 0}$ & 179686 & $0.985(0.982,0.987)$ & $<0.0001$ & \\
$\geq \mathbf{1 4 0}$ & 19749 & $0.992(0.988,0.997)$ & 0.0003 & \\
$\mathbf{D B P}$ & & & & $<0.0001$ \\
$<\mathbf{9 0}$ & 183594 & $0.984(0.982,0.986)$ & $<0.0001$ & \\
$\geq \mathbf{9 0}$ & 15841 & $0.999(0.994,1.005)$ & 0.8486 & \\
\hline
\end{tabular}

672 Note 1: Above model adjusted for gender, BMI, SBP, DBP, FPG, TC, TG, HDL-C, LDL-C, ALT, AST, family history of diabetes,

673 smoking and drinking status.

674 Note 2: In each case, the model is not adjusted for the stratification variable. 
Figures

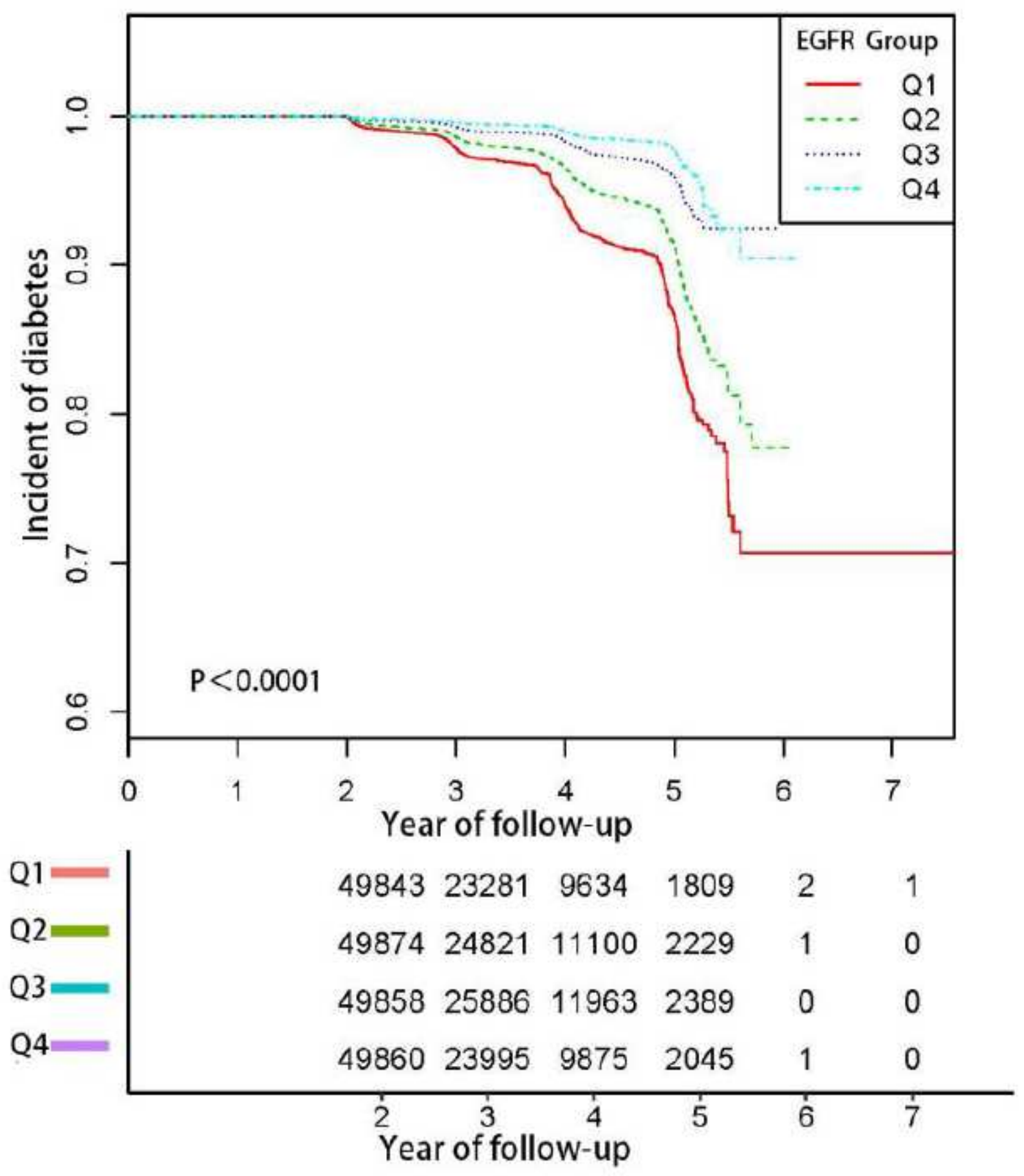

Figure 1

Kaplan-Meier event-free survival curve. Kaplan-Meier analysis of incident of diabetes based on eGFR quartiles (log-rank, $\mathrm{P}<0.0001)$. 


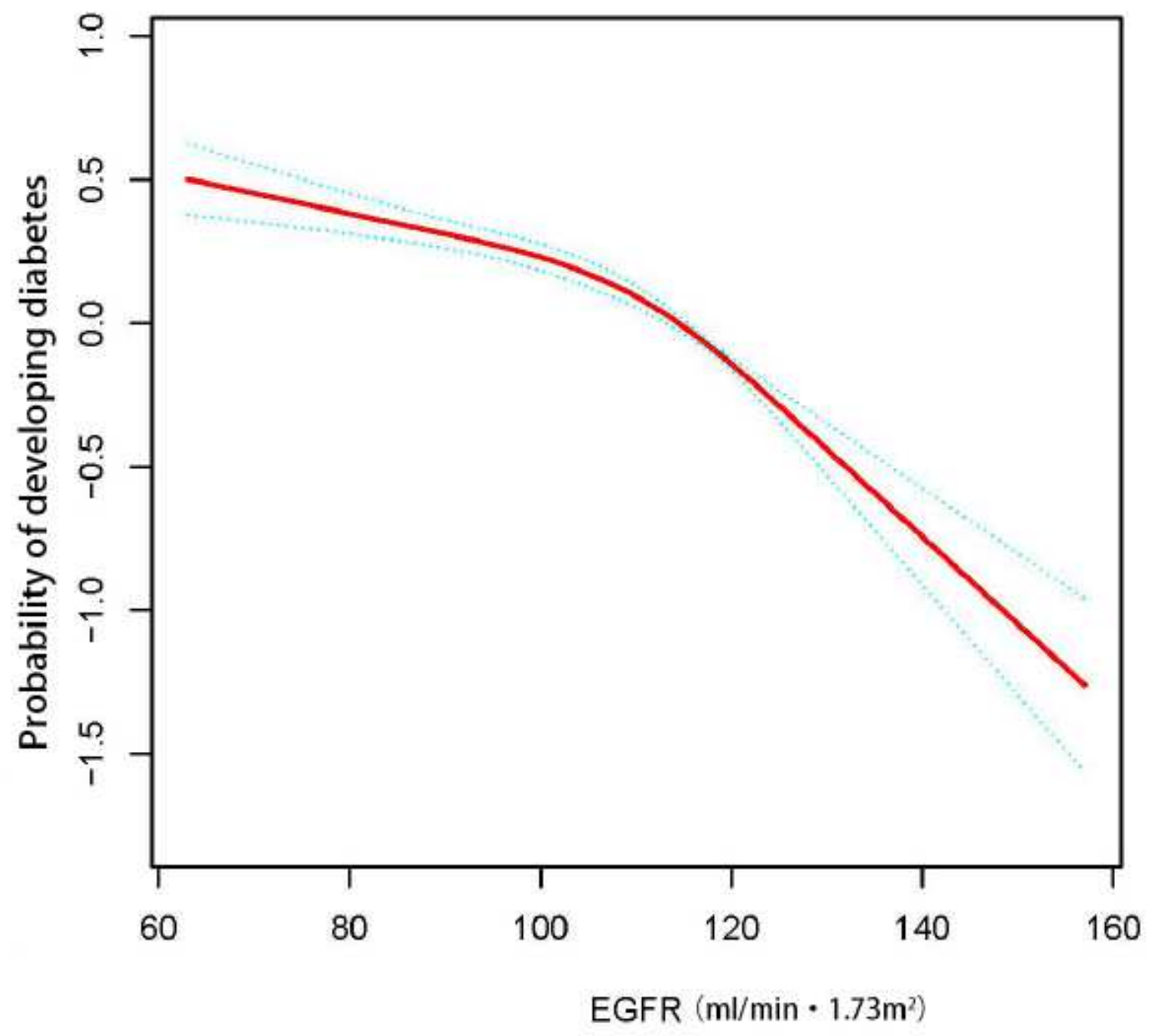

Figure 2

The non-linear relationship between eGFR and incident of diabetes. A non-linear relationship between them was detected after adjusting for gender, BMI, SBP, DBP, FPG, TC, TG, HDL-C, LDL-C, ALT, AST, family history of diabetes, smoking and drinking status. 